\title{
Book summary - The Process Matters
}

\section{Engaging and equipping people for success}

rockner, J. 2017. The Process Matters: Engaging and Equipping People for
Success. Princeton and Oxford: Princeton University Press. https://press. princeton.edu/titles/10432.html

Chapter 1 - Introduction: In this introductory chapter the author, Joel Brockner, sets out his aims for writing this book, the main point of which is that how doing things in the right manner (the process) can lead to important differences and better outcomes. It will also explore why things are not done in the right way and how to identify barriers and remove them from the process.

Brockner explains that he will provide a number of differing situations where two or more parties are involved and are trying to move towards a goal or complete an objective; the majority of these examples are centred in the workplace.

Chapter 2 - It's Only Fair: This chapter presents three scenarios showing the type of situations people come across in their jobs for the reader to consider. These scenarios and the solutions to them are based on fairness. From research undertaken in the 1970s, "we learned that people's perceptions of and reactions to fairness also depended upon the process accompanying the outcomes".

The author provides a table "Employees Reactions to Organizational Events and Decisions" which shows that the process has much more of an impact when the outcome is bad on favourability perception; if the outcome is good then the process has less of an impact. Additionally, it shows that when the process is fair, the outcome has less of an effect on employee's productivity and morale, relative to when they thought the process was unfair.

The next section of the chapter aims to clarify a few misconceptions:

- Fake it until you make it.

- Don't worry about the outcome as long as the process is fair.

- High process fairness can even get people to react quite positively to bad outcomes.

The advice for managers having to make tough decisions is: "they should plan and implement them with high process fairness, be aware of the fact that those on the receiving end of the unfavourable outcomes may blame themselves, and then help them to make selfblame attributions that are more behavioural and/or less characterological".

At the end of the chapter the author returns to the three scenarios presented at the beginning, concluding that employees will tend to be more loyal to their employer if the employer has demonstrated a high degree of fairness during the process.

Chapter 3 - Making Change Happen: It's All (or at least Largely) in the Process: This chapter focuses largely on organizational change; change generally provokes two different reactions in people: one of excitement and optimism, the other of resistance or even apathy. 
The author then examines some of the driving forces which prompt people to want to change, as well as the restraining forces which prevent the desire for change. It is stressed that a high quality change process is more likely to lead to employees supporting change and can be represented as such:

$$
\text { Change }=(\mathrm{D} \times \mathrm{V} \times \mathrm{P})>\mathrm{C}
$$

$\mathrm{D}$ stands for dissatisfaction with the present situation, $\mathrm{V}$ stands for the vision of the future, $\mathrm{P}$ stands for the process in place and $\mathrm{C}$ stands for the costs associated with the change.

Managers should provide as much explanation as to why the change is taking place as possible. This will encourage employees to be more accepting of the change. Uncertainty and negativity around impending change can snowball in organizations, so it is important for managers to get the company's "opinion leaders" on board at an early stage. The chapter then explains the importance of the change implementation plan, both at broad overview level and a more detailed, intricate view, and at regular intervals. It finishes by stressing the importance of open communication and active listening for leaders during the change process. These are vital to an organization in general but even more so during the change process.

Chapter 4 - Taking the Process Personally: This chapter begins by exploring the selfaffirmation processes and how they have aided morale and productivity amongst employees in the aftermath of a disruptive event, such as redundancies or site closures. Self-integrity allows us to evaluate the process in relation to the experience it creates for those on the receiving end.

The chapter then moves on to a case study of an on-boarding process at an organization that was intensified to not just focus on the company's culture and values, but those of the recent hires too. This "socialization" process not only increased employee morale but also reduced turnover rates. In addition, it can be more rewarding if self-affirmation processes occur not just when an employee starts with an organization but at future intervals too.

Next, the benefits of "job crafting" - where changes to job design come from the employees instead of management - are discussed. There are at least two reasons why job crafting increases feelings of self-affirmation; firstly, "when colleagues initiate job redesign the changes introduced are especially likely to be personally relevant. Second, the very fact that the change is employee initiated suggests that the change is employee initiated suggests that the process of job crafting allows people to experience a sense of control, in addition to the self-affirmation they may experience as a result of substantive changes to the job itself".

The chapter then gives examples of self-affirmation processes being used outside the workplace; such as high schools and colleges with some very encouraging results.

The chapter ends with some caveats on translating self-affirmation theory into practice:

- Coming from within.

- An end in its own right, not a means to an end.

- Do's and don'ts.

Chapter 5 - For Ethicality, the Process Also Matters: This chapter focuses on the importance of ethicality within a good-quality process. It emphasises that a poorly-planned process can be unethical itself (the ends justify the means) or that the negative impact of the process can cause employees to act unethically. "High process fairness is ethically correct. The importance people assign to their morality affects their reactions to how fairly they are treated".

Fairness in the process can be contagious and will be passed on by people in a number of ways; for example, paying it back and/or passing it down. However, this is also the case if a process is perceived to be unfair; those behaviours will likely be replicated and 
reciprocated. The next section of the chapter explores how global self-integrity - people seeing themselves as having identity, esteem and control - impacts on ethicality.

The chapter provides three examples where people have had to use some level of selfcontrol over emotions or behaviours for instance, but explains that enacting self-control can lead to ego depletion and reduce a person's ethicality, normally just in the short term. This supports the broader view that when people behave unethically, they generally aren't a bad person, but may do bad things because of a situation.

Chapter 6 - A High-Quality Process: Easier Said Than Done: This chapter examines some of the costs caused by doing the process well and what can be done to address them. Two of the main factors in a fair process are participation and explanation; managers can be reluctant to increase these two factors from fear of looking weak. However, withholding information and not providing explanations could cause more anger and even lead to legal action against companies that are downsizing, compared to those that are more honest and open. Using qualities such as compassion, empathy and sound emotional intelligence in general are still not seen as having a place in organizations, but these are the sort of qualities managers need to facilitate a good process.

The next section explores both what individuals can do and what organizations can do in terms of overcoming barriers. A table is featured entitled "Managers' Emotional Regulation Strategies When Delivering Bad News", and contains some examples managers can utilise, with four different strategies:

- Justifying one's actions.

- Quarantining emotion.

- Releasing emotion.

- Diverting attention.

Organizations can provide managers with training or guidance relating to changes, or prepare them by telling them what to expect during a process and to be realistic in terms of factors such as morale, engagement or obstacles.

Appendices: The book has eight appendices:

1. The Change Implementation Survey.

2. Scoring Guide for the Change Implementation Survey.

3. Measure of Regulatory Focus.

4. Measure of Work Regulatory Focus.

5. Measure of "Openers".

6. Allport-Vernon-Lindzey Values.

7. Measure of Moral Identity.

8. Measure of Emotional Reappraisal.

For instructions on how to order reprints of this article, please visit our website: www.emeraldgrouppublishing.com/licensing/reprints.htm

Or contact us for further details: permissions@emeraldinsight.com 